\title{
A novel method for controlling the surface morphology of polymeric membranes
}

\author{
Da-Ming Wang ${ }^{\mathrm{a}}$, Tian-Tsair Wu ${ }^{\mathrm{a}}$, Fung-Ching Lin ${ }^{\mathrm{b}}$, Jyun-Yuan Hou ${ }^{\mathrm{b}}$, Juin-Yih Lai ${ }^{\mathrm{b}, *}$ \\ ${ }^{a}$ Department of Chemical Engineering, National Taiwan University, Taipei 10617, Taiwan \\ ${ }^{\mathrm{b}}$ Membrane Research Laboratory, Department of Chemical Engineering, Chung Yuan University, Chung Li 32023, Taiwan
}

Received 4 August 1999; received in revised form 15 October 1999; accepted 15 October 1999

\begin{abstract}
It was found that, by the addition of appropriate hydrophilic nonsolvent in the hydrophobic polymer solution, hydrophobic polymeric membranes with 'cellular surface' structure can be fabricated. 'Cellular surface' is to denote the structure of the membranes having surface pores with a polygonal (nearly hexagonal) pattern. In the present work, the formation mechanism of the 'cellular surface' structure was investigated by visualizing the change on the surface of casting solution during membrane formation. When the hydrophobic polymer solution containing hydrophilic additives was exposed to air containing water vapor, the water vapor was drawn onto the casting solution and resulted in nucleation of emulsion drops spontaneously. The nuclei of emulsion drops grew, coalesced, and ended up with the cellular surface pores when the polymer was precipitated. With the knowledge of the formation mechanism of surface pores, the size surface pores can be tailored by controlling the growth of the nuclei of emulsion drops, either by adjusting the amount of hydrophilic additives or by altering the time period, allowing the nuclei to grow. (C2000 Elsevier Science B.V. All rights reserved.
\end{abstract}

Keywords: Membrane preparation and structure; Microporous and porous membranes; Surface morphology; Spontaneous emulsification

\section{Introduction}

Membrane techniques have been widely used to separate gaseous and liquid mixtures [1]. Different membrane separation processes require different membrane structures to bring about good separation performance [2]. Therefore, it is important to be able to fabricate membranes with different structures. Preparation of membranes with a variety of symmetric or asymmetric structures can be accomplished by using the phase inversion method [3]. In this method, adding nonsolvent in the casting solution is a con-

\footnotetext{
* Corresponding author. Tel.: +886-3-4563-672; fax: $+886-3-4563-672$.
}

venient and effective procedure to tailor membrane structure [4]. Control of the surface morphology of polymeric membranes by using nonsolvent additives is discussed in the present work.

It was reported [5] that membranes with 'cellular surface' structure can be fabricated by adding acetic acid in the casting solution of poly(4-methyl-1-pentene) (TPX) and cyclohexane. A typical 'cellular surface' structure is shown in Fig. 1. The characteristic of this structure is that surface pores with a polygonal (nearly hexagonal) pattern are embedded on the membrane top surface. The major aim of the present work is to systematically investigate the formation mechanism of the 'cellular surface' structure and to show that the size of the surface pores is controllable. 


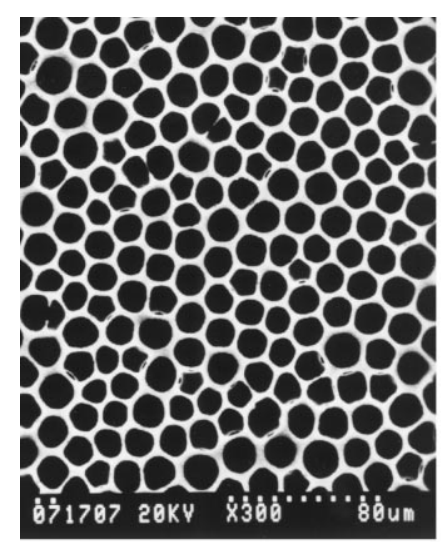

(A)

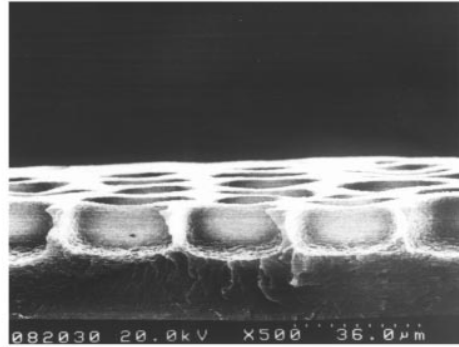

(B)

Fig. 1. Scanning electron micrographs for a typical 'cellular surface' structure. (A) surface; (B) cross-section.

Similar polygonal patterns on surface have also been observed in systems such as Bernard-flow [6], evaporation of paint [7], oil recovery [8] and the formation of foam [6]. It will be shown in the present work that the formation of 'cellular surface' structure is closely related to the phenomenon of spontaneous emulsification [9], which has been used to explain the formation of polygonal patterns in the system of oil recovery [8]. When two immiscible fluid is brought together, it requires energy, such as mechanical agitation, to form an emulsion [10]. However, when a third component, miscible with the two fluids, is added in one phase, spontaneous emulsification can be observed. It is believed that the diffusion caused by the addition of the third component can produce regions of local supersaturation and consequently results in nucleation of emulsion drops [11]. Spontaneous emulsification has been applied to liquid-liquid extraction [11], oil recovery [8], and drug encapsulation [12]. In addition, the phenomenon of spontaneous emulsification was used to account for the formation of macrovoids in polymeric membranes [13].

In this work, it will be shown that the water vapor in air plays a very important role in the formation of cellular surface pores. It has been reported that, when water vapor was drawn into the polysulfone casting solution, membranes with porous surface [14] and with porous cross-section [15] can be successfully fabricated. The polymer solvents used in $[14,15]$ are dimethylformamide and $n$-methyl-2-pyrrolidone, having high affinity for water. Therefore, water vapor can be easily absorbed into the polymer solution, initiate phase separation, and result in surface pores. However, the solvent used in our study is cyclohexane, a hydrophobic solvent which cannot draw water into the casting solution. In such a hydrophobic casting environment, hydrophilic additives, such as acetic acid, is required to draw water vapor into the solution to induce spontaneous emulsification and initiate the formation of 'cellular surface' structure. The growth and coalescence of these emulsion drops would result in cellular surface pores when polymer is precipitated. The details of the formation mechanism and how to control the size of surface pores are presented in the following.

\section{Experimental}

\subsection{Materials}

The poly(4-methyl-1-pentene)(TPX, MX-002) used in this study was supplied by Mitsui company. Poly(ethylene-co-vinyl acetate) (EVA, $14 \mathrm{wt} . \%$ vinyl acetate) was purchased from Aldrich Co. and polystyrene (PS) was synthesized following the procedure of [16]. All polymer solvents were purchased from Merck Co. and all additives were of reagent grade. The chemicals were used without further purification. 


\subsection{Membrane preparation}

Polymer was dissolved at $60^{\circ} \mathrm{C}$ in a solution of polymer solvent and additive to form a $4.8 \mathrm{vol} \%$ of polymer solution. The polymer solution was kept at $40^{\circ} \mathrm{C}$ for $24 \mathrm{~h}$ and then cast on a glass plate to a predetermined thickness with a Gardner knife. The casting solution was evaporated in air for an hour to precipitate the polymer, then the resulted membrane was peeled off from the glass plate in water. For some cases, the casting solution was evaporated in the air for $30 \mathrm{~s}$ or an hour, and was then immersed in a coagulation bath to precipitate the polymer.

\subsection{SEM}

The membrane structure was examined by a Hitachi (Model S4000) scanning electron microscope (SEM). In SEM studies, membrane samples were fractured in liquid nitrogen and coated with platinum.

\subsection{Optical studies}

A transparent glass cell was used to observe the change on the surface of casting solution during membrane formation. The set-up is shown in Fig. 2. The casting solution was put in the bottom of the cell and air was then introduced into the cell slowly. We can adjust the flow-rate ratio of the dry and water-saturated air to control the humidity of the introduced air. A CCD camera (Mitsubishi Chemical, VS 90) with a magnification of 100 was used to videotape the change on the solution-air interface during evaporation.

\subsection{Total volume of surface pores}

Since the membrane consists of two parts: polymer and surface pores, the volume of surface pores can be determined by subtracting the volume occupied by polymer from the membrane volume. After the area $(A)$, the mass $\left(W_{\mathrm{m}}\right)$ and the thickness $(D)$ of a membrane sample were measured, the volume of the membrane sample $\left(V_{\mathrm{m}}\right)$ can be calculated by $D \times A$ and the volume occupied by polymer $\left(V_{\mathrm{p}}\right)$ can be expressed as $W_{\mathrm{m}} / \rho_{\mathrm{p}} . \rho_{\mathrm{p}}$ is the density of polymer and has a value of $0.838 \mathrm{~g} / \mathrm{cm}^{3}$ for TPX. Then, the volume of surface pores can be estimated by the following equation:

Volume of surface pores $=V_{\mathrm{m}}-V_{\mathrm{p}}=D \times A-\frac{W_{\mathrm{m}}}{\rho_{\mathrm{p}}}$

It should be noted that the above equation in fact calculates the total volume of all pores. For the membranes prepared by the dry method, the pores only existed near the surface and the bulk is dense. Therefore, the results from the above equation can be used to estimate the volume of surface pores. The above procedure was used to estimate the volume of surface pores for a homogeneously dense membrane. For the dense membrane, the ideal value is zero and the measured value is also very close to zero, indicating that the above procedure is reliable.

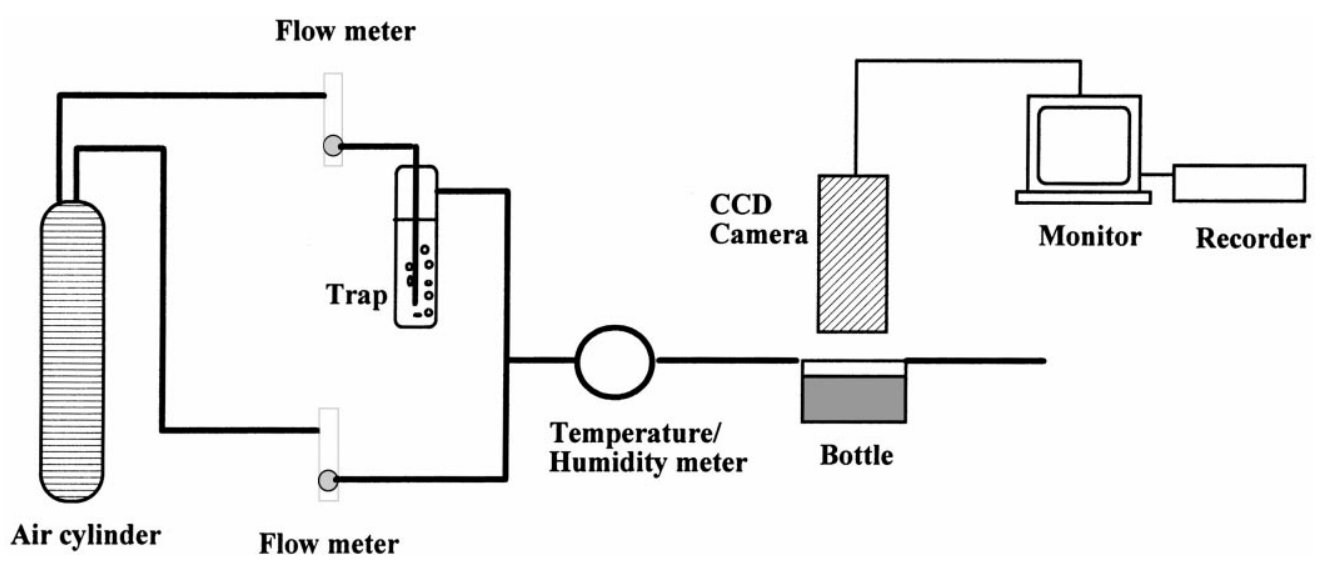

Fig. 2. Optical apparatus for observing the formation of 'cellular surface' structure. 


\subsection{Water tolerance}

In the present work, water tolerance of a system is defined as the volume of water required to bring the system to phase separate. Generally speaking, lower water tolerance indicates that the system is easier to phase separate when water is introduced. The water tolerance of a solution was determined by the titration method described below. A mixture of solvent and additive was stirred in a glass tube to form a clear solution for titration. Distilled water was added in the solution until the solution became turbid, representing the cloud point had been reached. The volume of the added water was recorded as the water tolerance. It should be noted that the experiment was performed at $25^{\circ} \mathrm{C}$.

\section{Results and discussion}

\subsection{Optical study of the formation of cellular surface pores}

It has been reported that membranes with cellular surface pores (Fig. 1) can be fabricated by evaporating the polymer solution, containing $4.8 \mathrm{vol} \%$ of TPX, 80.2 vol\% of cyclohexane and 15 vol\% of acetic acid [5]. Since the solvent cyclohexane has higher volatility than the nonsolvent acetic acid, the nonsolvent/solvent ratio increases during the evaporation stage. Therefore, one might suspect that the increasing nonsolvent/solvent ratio during evaporation causes phase separation (demixing) in the casting solution and generates the surface pores. However, the formation mechanism is not so simple. A phenomenon which cannot be explained by the above mentioned mechanism is that the surface pores cannot be observed when the casting solution was evaporated in dry air (without water vapor). A more appropriate mechanism to account for the formation of cellular surface pores is given below.

First, the importance of water vapor in the formation of cellular surface pores is discussed. We used the device shown in Fig. 2 to adjust the content of water vapor in air during membrane formation. It was found that, when the relative humidity in air was $15 \%$, the surface pores cannot be observed (see Fig. 3A). The structure of surface pores for a relative humidity of $60 \%$ is presented in Fig. 1A and the resulted membrane structure for $95 \%$ relative humidity is shown in Fig. 3B. Obviously, the formation of surface pores is strongly related to the content of water vapor in air. In order to understand more clearly about the formation mechanism of the cellular surface pores, an optical set-up (Fig. 2) was used to observe the evolution of the solution-air interface during membrane formation.

The alteration of interface was recorded by a video camera and the results are shown in Fig. 4. Nucleation of emulsion drops can be observed about $7 \mathrm{~s}$ after the exposure of casting solution (TPX/cyclohexane/acetic acid) to water-saturated air. The nuclei of emulsion drops grow during membrane formation. With an evaporation time of about $21 \mathrm{~s}$, the emulsion drops begin to contact each other. The contact and squeeze

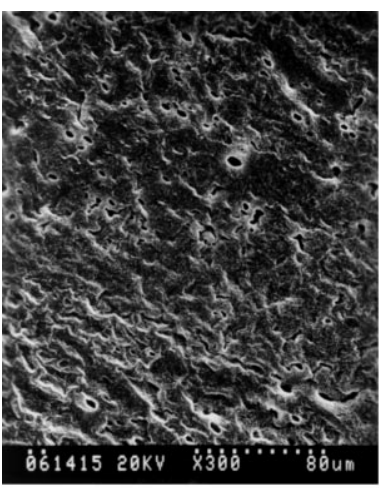

(A)

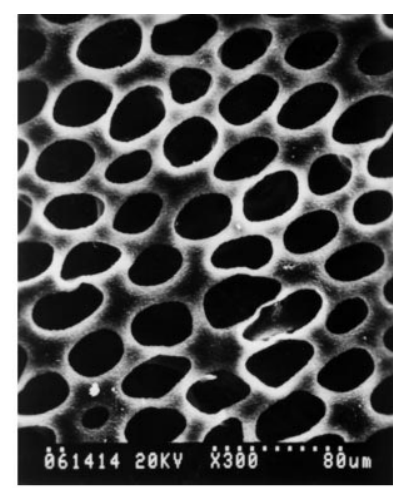

(B)

Fig. 3. Effect of humidity in air on the formation of surface pores. (A) Relative humidity: 15\%; (B) Relative humidity: $95 \%$. 


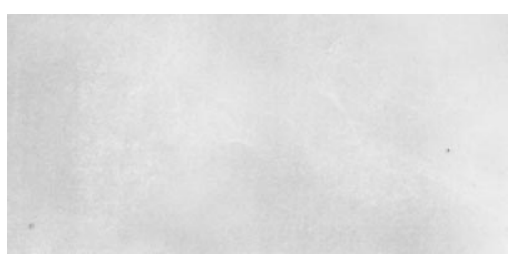

(A)

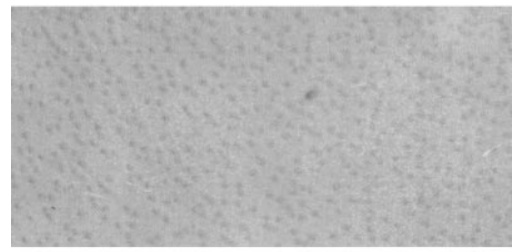

(B)

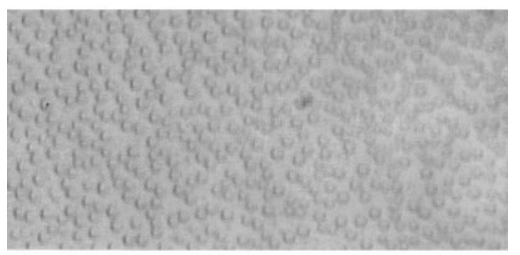

(C)

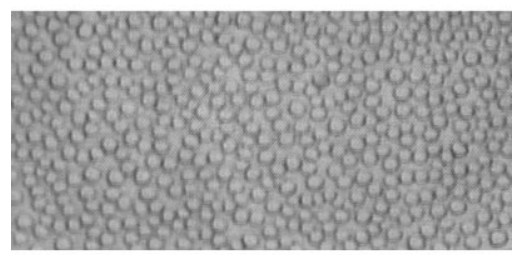

(D)

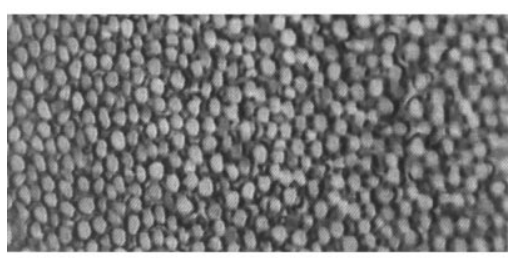

(E)

Fig. 4. Evolution of the surface of casting solution during the formation of membranes with cellular surface pores. (A) $0 \mathrm{~s}$; (B) $7 \mathrm{~s}$; (C) $9 \mathrm{~s}$; (D) $21 \mathrm{~s}$; (E) $52 \mathrm{~s}$.

of the emulsion drops can form a stable polygonal cell pattern which is the origin of the 'cellular surface' structure. After $21 \mathrm{~s}$, coalescence of the emulsion drops is also observed.

The above results suggest that the formation of cellular surface structure be originated from the spontaneous emulsification induced by the contact of casting solution with water vapor. The water vapor in air plays a crucial role in the spontaneous emulsification, evidenced by the fact that no emulsification drops were observed when the casting solution was evaporated in dry air. On the other hand, the added acetic acid is also very important because the contact of water vapor with the solution of TPX/cyclohexane (without acetic acid) could not generate spontaneous emulsification. An explanation for the above observation is given below. The casting solution (TPX/cyclohexane) is very hydrophobic; therefore, phase separation occurs to form nuclei of emulsion drops when water vapor is drawn onto the casting solution. When the air does not contain water vapor, no water can be drawn to the solution to initiate the spontaneous emulsification. On the other hand, TPX/cyclohexane solution alone is too hydrophobic to draw water to form surface pores. The hydrophilic additive (acetic acid in this case) is required to draw water vapor onto the casting solution to initiate spontaneous emulsification.

On basis of the above information, a mechanism for the formation of 'cellular surface' structure is proposed. The addition of acetic acid (hydrophilic additive) in the TPX/cyclohexane solution (hydrophobic casting solution) can draw water from air into the casting solution. The introduction of water can generate spontaneously regions of supersaturation and nucleation of emulsion drops. These emulsion nuclei would grow and coalesce during the evaporation stage and result in cellular surface pores when the polymer is precipitated.

The observation that the 'cellular surface' structure can be formed by the growth of emulsion drops is similar to that foams or cellular emulsions can be made by increasing the volume fraction of the dispersed phase [6]. The new idea presented in this work is the method of introducing the dispersed phase. The dispersed phase in this work is a combination of the water vapor in air and the hydrophilic additive (acetic acid). The hydrophilic additive was introduced into the polymer solution first but did not induce phase separation. Therefore, homogeneous casting solution could be obtained. The formation of the dispersed phase was initiated by the water vapor in air and the growth of the dispersed phase was controlled by the amount of the additive (will be discussed later). It should be noted that homogeneous casting solution 
could not be obtained and poor membrane formation was observed when water was directly introduced into the polymer solution.

\subsection{Role of polymers in the formation of cellular surface pores}

According to the discussion in the preceding section, it is known that the spontaneous emulsification, induced by drawing water vapor into the casting solution, is responsible for the occurrence of 'cellular surface' structure. The casing solution consists of polymer (TPX), solvent (cyclohexane), and additive (acetic acid). In this section the role of polymer is discussed.

According to Fig. 5, surface pores can still be found when TPX was replaced with polystyrene (PS), ethylene-co-vinyl acetate (EVA), and the blend of TPX (50 wt.\%) and EVA (50 wt.\%), suggesting that the occurrence of surface pores is insensitive to the polymer used. Since the surface pores are resulted

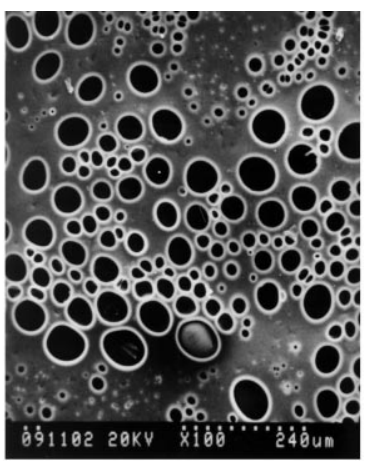

(A)

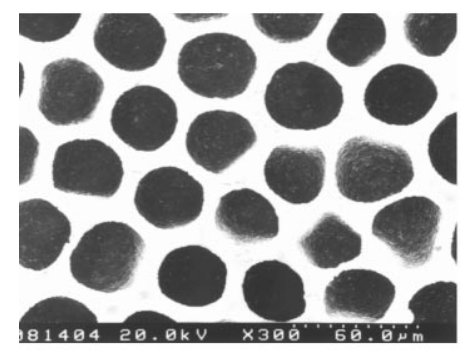

(C) from the spontaneous emulsification induced by water vapor, the above observation indicates that the type of polymer is not an important variable in determining if spontaneous emulsification can occur. Therefore, it is expected that spontaneous emulsification can as well be observed on the surface of a solution containing only the solvent and additive but without polymer. This expectation was verified and the result is shown in Fig. 5D.

The above results suggest that, if the spontaneous emulsification can be observed in a solution, membranes with cellular surface pores can then be prepared by dissolving polymers in this solution, and vice versa. This deduction has been verified by investigating the spontaneous emulsification in several systems containing different solvents and additives. As shown in Table 1, for those solutions that spontaneous emulsification can be observed, cellular surface pores can also be observed when polymer was dissolved in these solutions. For those not, cellular surface pores cannot be found either.

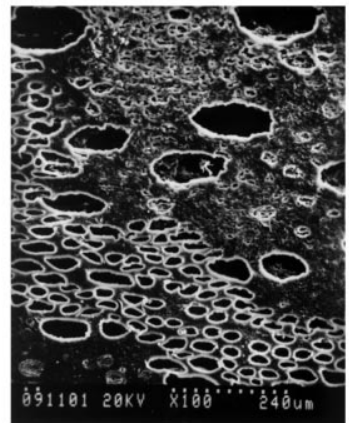

(B)

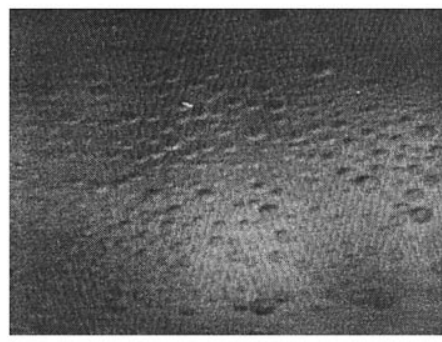

(D)

Fig. 5. Effect of the types of polymer on the formation of membranes with surface pores. (A)PS; (B) EVA; (C) TPX/EVA blend (1:1); (D) No polymer, Temperature: $28^{\circ} \mathrm{C}$; Relative humidity: $75 \%$. 
Table 1

Formation of cellular surface pores or surface emulsion drops in different systems

\begin{tabular}{llll}
\hline Solvent & Additive & Polymer & Result \\
\hline Cyclohexane & Acetic acid & TPX & $+^{\mathrm{b}}$ \\
Cyclohexane & Acetic acid & PS & + \\
Cyclohexane & Acetic acid & EVA & + \\
Cyclohexane & Acetic acid & $*$ a & + \\
Cyclohexane & NMP & TPX & + \\
Cyclohexane & NMP & $*$ & + \\
Cyclohexane & DMAc & TPX & + \\
Cyclohexane & DMAc & $*$ & + \\
Methylcyclohexane & Acetic acid & TPX & + \\
Methylcyclohexane & Acetic acid & $*$ & + \\
Cyclohexane & Propionic acid & TPX & $-{ }^{\mathrm{b}}$ \\
Cyclohexane & Propionic acid & $*$ & - \\
Cyclohexane & Acetone & TPX & - \\
Cyclohexane & Acetone & $*$ & - \\
Cyclohexane & Ethanol & TPX & - \\
Cyclohexane & Ethanol & $*$ & - \\
Cyclohexane & 1-Butanol & TPX & - \\
Cyclohexane & 1-Butanol & $*$ & - \\
Toluene & Acetic acid & PS & - \\
Toluene & Acetic acid & $*$ & - \\
\hline
\end{tabular}

a $*$ : No polymer.

$\mathrm{b}+,-$ : with, without surface pores or emulsion drops.

A possible explanation is given below to account for the above observation. It was noticed that, for those systems that cellular surface pores can be formed, the fluidity of the casting solution near the interface was much higher than that in the bulk during evaporation. Because the hydrophobic polymer has much lower affinity for water vapor than the hydrophilic additive, the additive has higher tendency than the polymer to move toward the solution-air interface when the solution is in contact with water vapor. Therefore, the polymer concentration near the interface could be lower than that in the bulk. The low polymer concentration near the interface can explain the high fluidity near the interface. Since the polymer concentration is low near the interface, the interfacial property is not much affected by the property of polymer.

Although the occurrence of surface pores is insensitive to the polymer used, the types of polymer can have effect on the morphology of surface pores, as shown in Fig. 5. Whether water vapor can be drawn onto the casting solution or not could only be affected by the interfacial property and is therefore, not sensitive to the types of polymer. However, the growth of emulsion nuclei to form surface pores is not an interfacial phenomenon. Therefore, the morphology of surface pores is different for different polymers.

\subsection{Role of solvents and additives in the formation of cellular surface structure}

In Table 1 we present several solvent-additive pairs that can produce spontaneous emulsification and fabricate membranes with cellular surface pores and several other pairs that cannot. A possible mechanism for the spontaneous emulsification is the local supersaturation induced by mass transfer [11]. According to the mechanism of local supersaturation generated by mass transfer, the thermodynamic property such as the binodal curve is very important [11].

In the present work, the binodal (coexistence) curve of the water-solvent-additive ternary system was determined by titrating water into the solution of solvent-additive till the onset of turbidity, indicative of the occurrence of phase separation. The results (Fig. 6) show that systems with spontaneous emulsification have less water tolerance than those systems without spontaneous emulsification. This observation can be accounted for by that, when water vapor is drawn to the solution, systems with less water tolerance are easy to phase separate and result in spontaneous emulsification.

On basis of the above results, it seems that water tolerance can be used as a criterion to predict if spontaneous emulsification and cellular surface pores can occur. However, it should be noted that thermodynamic property alone is not enough to completely account for the occurrence of spontaneous emulsification. In fact, the condition required for spontaneous emulsification is an intersection between the diffusion path line and the binodal curve [11]. Therefore, for complete analysis of the formation of cellular surface pores, investigation on mass transfer, which still remains a challenge, should also be carried out.

\subsection{Control of the size of surface pores}

It was found that the size of surface pores is dependent on the amount of additives. The results shown in 


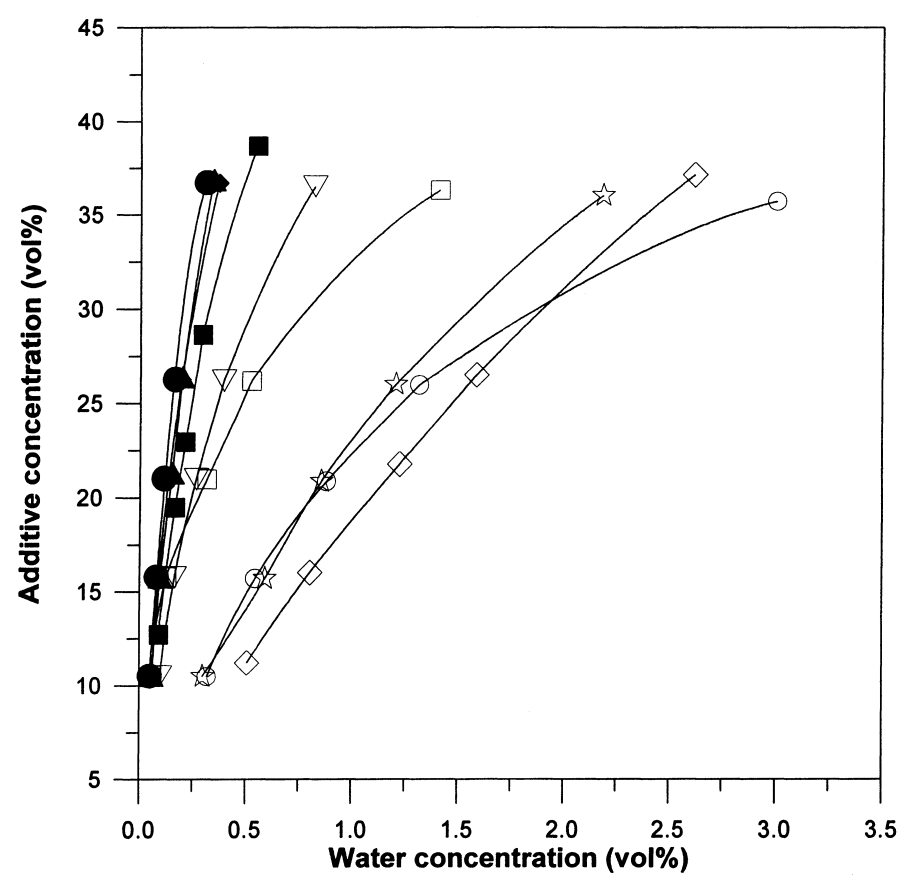

Fig. 6. Water tolerance for different polymer solvent-additive systems. ( $)$ Cyclohexane-NMP; ( $\mathbf{\Delta})$ Cyclohexane-DMAc; ( $)$

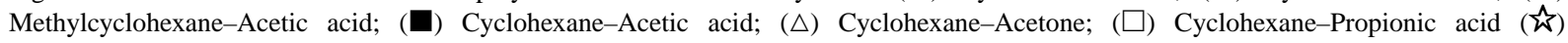
Cyclohexane-Butanol; $(\diamond)$ Cyclohexane-Ethanol; $(\bigcirc)$ Toluene-Acetic acid. Solid symbols represent the systems that can produce 'cellular surface' structure and open symbols represent those cannot.

Fig. 7 suggest that the size of surface pores varies with the concentration of the additive (acetic acid). When the volume fraction of acetic acid is less than $10 \mathrm{vol} \%$, the size of surface pores increases with increasing concentration of acetic acid. When the concentration of acetic acid is higher than $15 \mathrm{vol} \%$, there is no obvious change in the size of surface pores; but the shape becomes irregular. In addition, when the concentration of acetic acid is higher than $15 \mathrm{vol} \%$, the pores are not limited to a single layer but form a mutilayer structure as shown in Fig. 8.

It should be noted that, when we adjusted the concentration of acetic acid, the concentration of cyclohexane was changed but the concentration of TPX was fixed (4.8vol\%). By keeping fixed polymer concentration, the viscosity and gelation of polymer solution should not change much when the concentration of acetic acid was varied. Therefore, the effect of the amount of acetic acid can be clarified without being complicated by the effect of gelation time.
By visualizing the formation of surface pores, it was found that the growth of the nuclei of emulsion drops is strongly dependent on the concentration of the added acetic acid. The growth of nuclei is limited at lower concentration of acetic acid and the nuclei can grow larger when the concentration of acetic acid is higher. When the concentration of acetic acid is higher than $15 \mathrm{vol} \%$, the growth of nuclei would make them contact each other and coalescence of emulsion drops can be observed. In the same time when coalescence was observed, another layer of emulsion drops began to occur and grow beneath the surface layer. The above observation suggests that the growth of emulsion nuclei, which determines the size of surface pores, be controlled by the amount of acetic acid (hydrophilic additive).

To discriminate which one is the determining factor for the size of surface pores: the concentration or the total amount of acetic acid, we investigated the effect of the thickness of casting film on the size of 


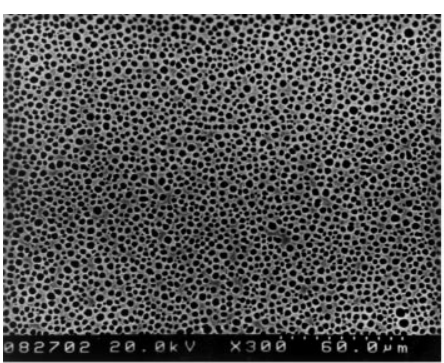

(A)

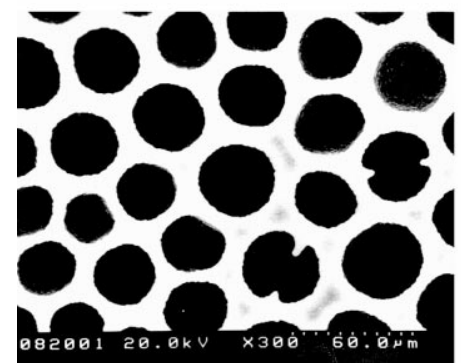

(C)

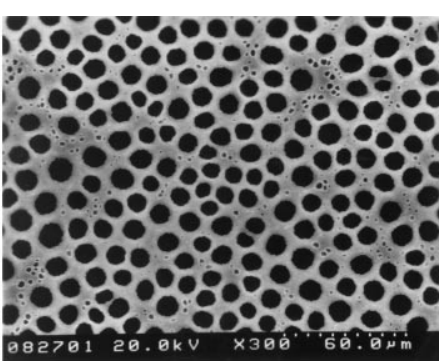

(B)

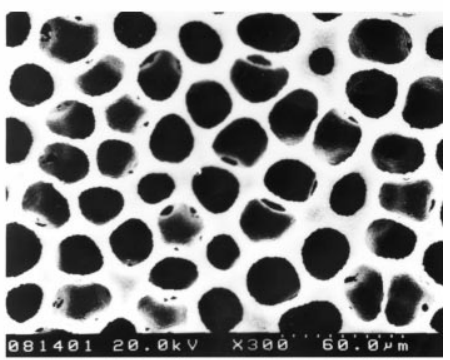

(D)

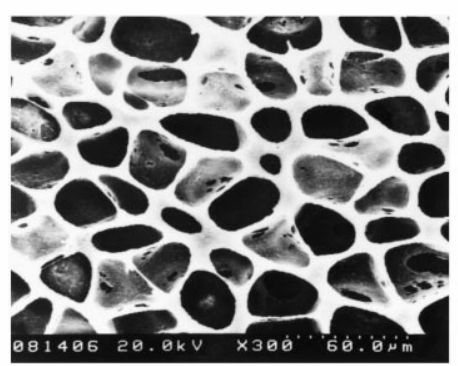

(E)

Fig. 7. Effect of the concentration of acetic acid on the surface structure of membranes. (A) $2.5 \mathrm{vol} \%$; (B) $5 \mathrm{vol} \%$; (C) $10 \mathrm{vol} \%$; (D) $15 \mathrm{vol} \%$; (E) $20 \mathrm{vol} \%$. Temperature: $28^{\circ} \mathrm{C}$; Relative humidity: $65 \%$; Casting thickness: $500 \mu \mathrm{m}$.

surface pores. When the thickness of casting film increased but the concentration of acetic acid was kept fixed, the total amount of acetic acid also increased because of the increase in the volume of casting solution. It can be seen from Fig. 9 that the increase in casting thickness can make the surface pore larger and can sometimes make the pore structure become multilayer. These results indicate that, by keeping the concentration of acetic acid fixed, an increase of the total amount of acetic acid can still make the emulsion drops grow larger and result in larger surface pores.
We have also investigated the relationship between the total volume of surface pores and the concentration of acetic acid for three different thickness of casting: $300,500,600 \mu \mathrm{m}$. The total volume of the surface pores was determined by the method presented in Section 2.5. When we plotted the volume of surface pores versus the concentration of acetic acid, it was found that, with the same concentration of acetic acid, membranes with different casting thickness possessed different pore volume (Fig. 10). However, when we plotted the pore volume versus the total amount of acetic acid, all the data can be well fitted into a single 


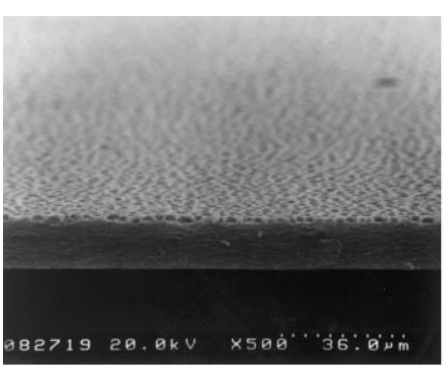

(A)

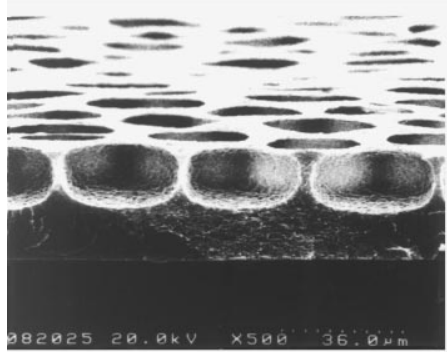

(C)

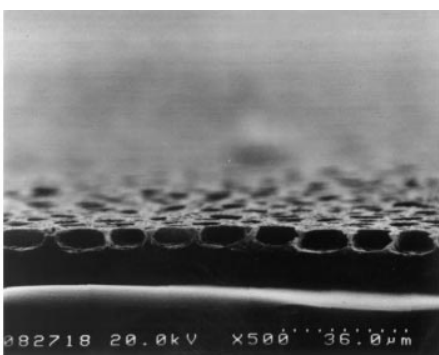

(B)

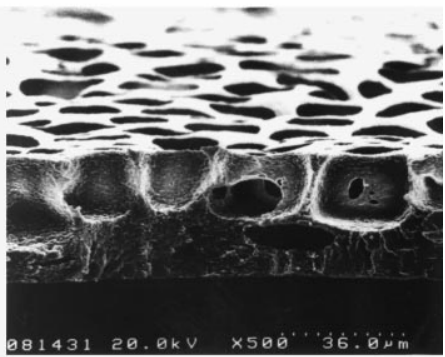

(D)

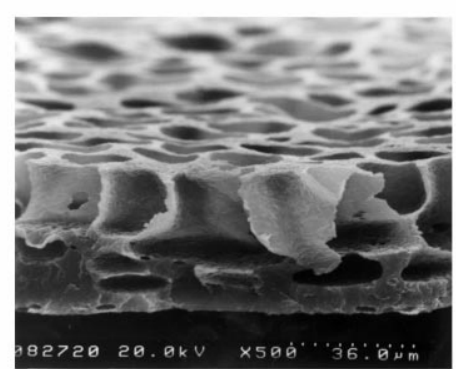

(E)

Fig. 8. Effect of the concentration of acetic acid on the cross-sectional structure of membranes. (A) $2.5 \mathrm{vol} \%$; (B) $5 \mathrm{vol} \%$; (C) $10 \mathrm{vol} \%$; (D) $15 \mathrm{vol} \%$; (E) $20 \mathrm{vol} \%$. Temperature: $28^{\circ} \mathrm{C}$; Relative humidity:65\%; Casting thickness: $500 \mu \mathrm{m}$.

curve as shown in Fig. 11. This observation indicates that the amount of acetic acid in the casting solution is the determining factor. In addition, since the volume of pores is determined by the growth of the nuclei of emulsion drops, it can be concluded that the growth of emulsion nuclei is controlled by the amount of acetic acid in the casting solution.

Another method to control the size of surface pores is to interfere the growth of emulsion drops by immersing the casting film in a coagulation bath. When the membrane is prepared by simply evaporating the casting solution in air containing water vapor, the nuclei resulted from spontaneous emulsification have enough time to grow and coalesce. On the other hand, when the casting solution is immersed in a coagulation bath after evaporating for a certain period of time, there is not enough time for the nuclei to grow and coalesce. Therefore, the resulted surface pores would be smaller. Fig. 12A and $\mathrm{C}$ are the surface and cross-sectional structures for a membrane prepared by evaporating the solution of TPX/cyclohexane/acetic acid for $30 \mathrm{~s}$ and then immersing the solution in a bath of ethanol (coagulant). Fig. 12B and D are the corresponding structures for a membrane prepared by evaporating the same solution for an hour without the immersion step. It is obvious that the size 


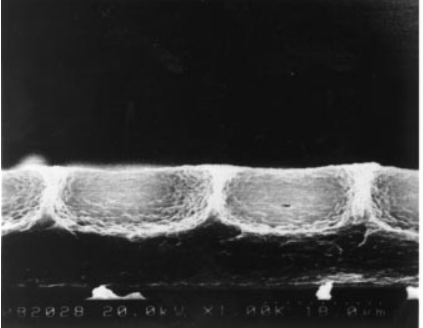

(A)

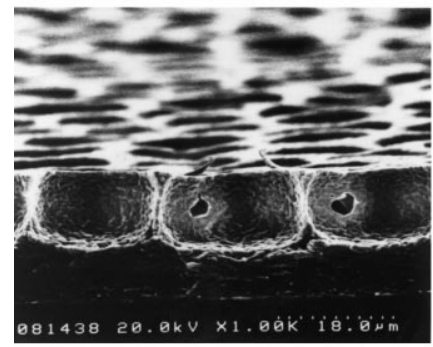

(C)

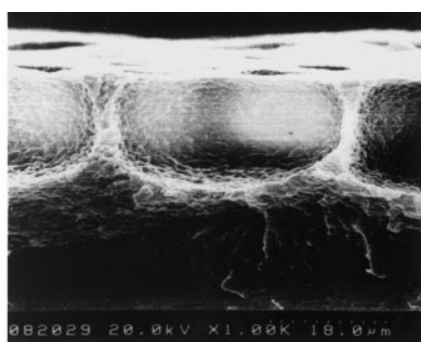

(B)

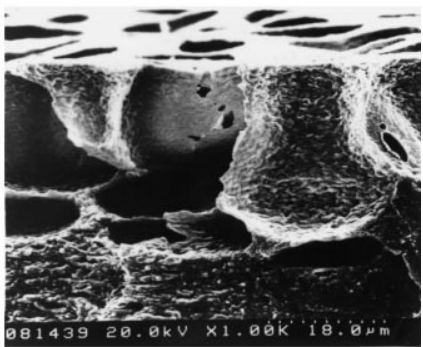

(D)

Fig. 9. Effect of casting thickness on the size of cellular surface structure. (A) $300 \mu \mathrm{m}$, acetic acid: 10 vol\%; (B) $600 \mu \mathrm{m}$, acetic acid: $10 \mathrm{vol} \%$; (C) $300 \mu \mathrm{m}$, acetic acid: $15 \mathrm{vol} \%$; (D) $600 \mu \mathrm{m}$, acetic acid: $15 \mathrm{vol} \%$. Temperature: $31^{\circ} \mathrm{C}$; Relative humidity: $60 \%$.

of surface pores is dependent on the evaporation time.

Adjusting the humidity of air can also be used to control the size of surface pores. As shown in Figs. 1

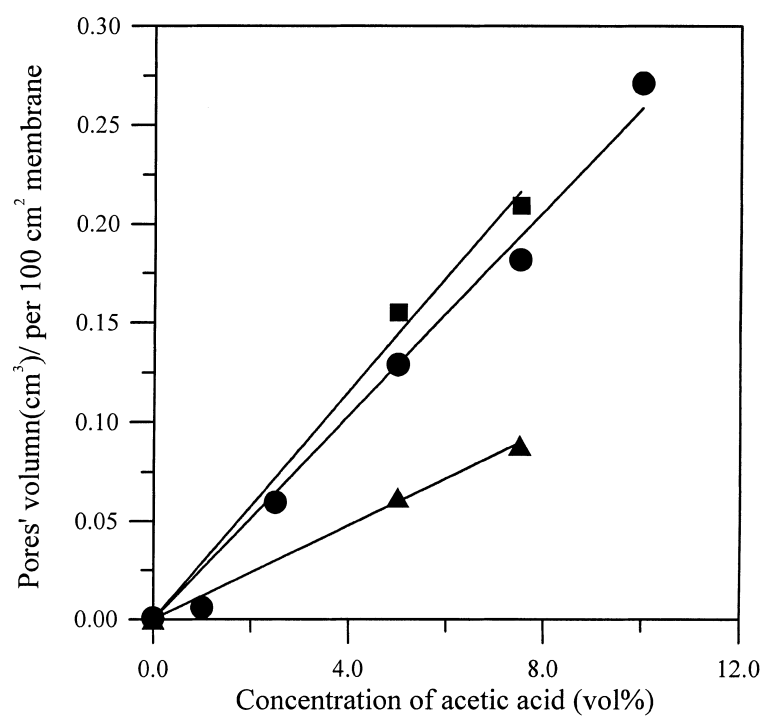

Fig. 10. Relationship between the volume of surface pores and the concentration of acetic acid for different thickness of casting

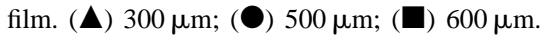

and 3 , the size of surface pores is different when the air humidity is changed. However, it is much easier to control the amount of additives and the evaporation time than to control the humidity in casting environ-

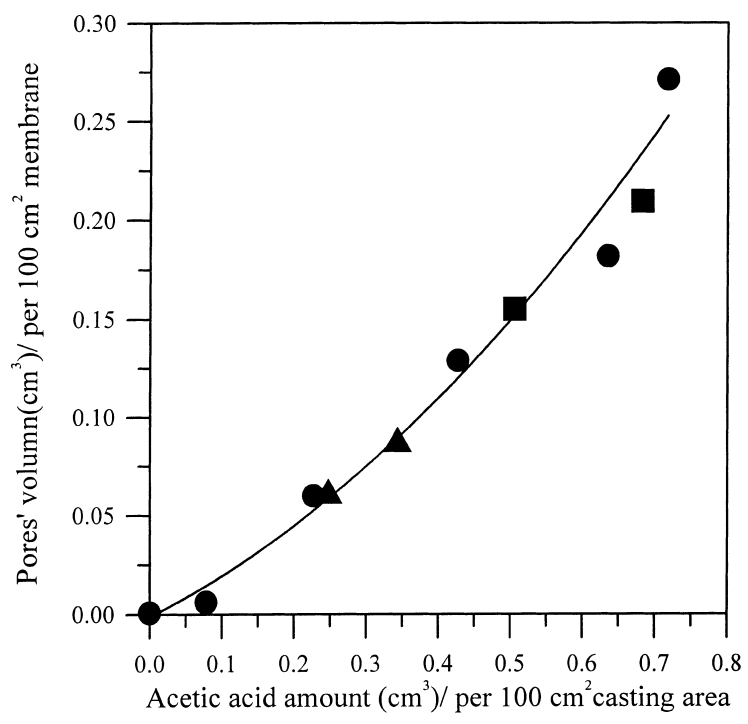

Fig. 11. Relationship between the volume of surface pores and the amount of acetic acid for different thickness of casting film.

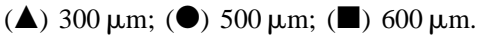




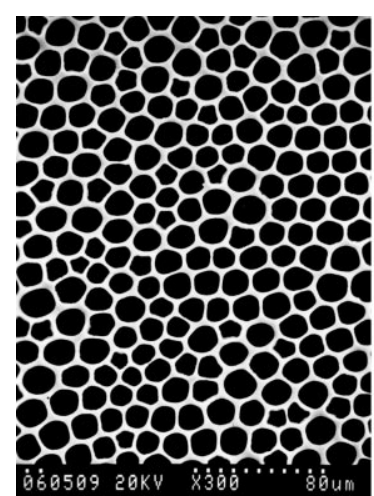

(A)

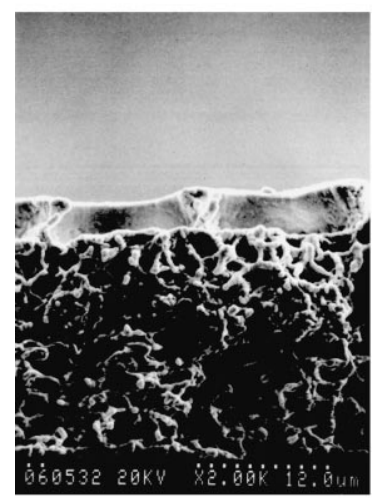

(C)

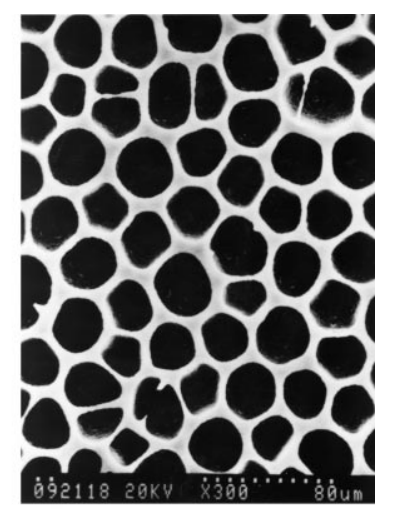

(B)

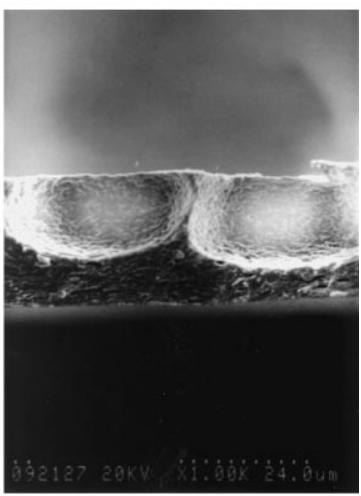

(D)

Fig. 12. Effect of evaporation time on the size of cellular surface pores. Surface: (A) $30 \mathrm{~s}$; (B) $1 \mathrm{~h}$, Cross-section: (C) $30 \mathrm{~s}$; (D) $1 \mathrm{~h}$.

ment. Therefore, detail investigation on the effect of air humidity on surface pores was not performed in the present work.

\subsection{Potential application of membranes with cellular surface pores}

Membranes with cellular surface pores could have several applications. First, because the morphology of such a membrane is different from that of normal membranes, the separation performance could be different. Second, it has been pointed out [17] that membranes with skin on one side and capillary pores on the other side, similar to the 'cellular surface' structure, could be useful to immobilize fungi for biocatalysis. In addition, it is known [18] that the morphology, especially the three-dimensional structure, of the materials used for cell culture can have dramatic influence on the growth rate and function of the cultured cells. Since the size of surface pores is controllable as described in the previous section, membranes with cellular surface pores could be useful for cell culture and could also be used to investigate the effect of surface morphology on cultured cells.

\section{Conclusions}

The formation mechanism of membranes with cellular surface pores has been elucidated in the present work. The evolution of the surface of casting solution during membrane formation was visualized by using optical instruments. When the hydrophobic casting solution containing hydrophilic additives was exposed to air containing water vapor, the water vapor could be drawn onto the casting solution and resulted in nucle- 
ation of emulsion drops spontaneously. The nuclei of emulsion drops would grow, coalesce and end up with cellular surface pores when the polymer was precipitated. It is also known that the occurrence of surface pores is insensitive to the types of polymer. For the systems we studied, it seems that systems with less water tolerance are inclined to produce cellular surface pores. In addition, the size of surface pores is determined by the growth of the nuclei of emulsion drops, which can be controlled by the amount of the hydrophilic additives and the time period allowing for the nuclei to grow. With this information, membranes with cellular surface pores can be fabricated by using different polymers and the size of cellular surface pores can be tailored.

\section{Acknowledgements}

The authors wish to sincerely thank the $\mathrm{Na}$ tional Science Council of Taiwan, ROC(NSC 89-2216-E-033-002) for the financial support of this project.

\section{References}

[1] R.E. Kesting, Synthetic Polymeric Membranes, Wiley, New York, 1985.

[2] R.E. Kesting, The four tiers of structure in integrally skinned phase inversion membranes and their relevance to the various separation regimes, J. Appl. Polym. Sci. 41 (1990) 2739.

[3] M. Mulder, Basic Principle of Membrane Technology, Kluwer Academic Publisher, London, 1991.

[4] P. Radovanovic, S.W. Thiel, S.T. Hwang, Formation of asymmetric polysulfone membranes by immersion precipitation Part II: The effects of casting solution and gelation bath compositions on membrane structure and skin formation, J. Membr. Sci. 65 (1992) 231.
[5] J.Y. Lai, F.C. Lin, C.C. Wang, D.M. Wang, Effect of nonsolvent additives on the porosity and morphology of asymmetric TPX membranes, J. Membr. Sci. 118 (1996) 49.

[6] C.A. Miller, P. Neogi, Interfacial Phenomena: Equilibrium and Dynamic Effects, Marcel Dekker, New York, 1985.

[7] C.M. Hansen, P.E. Pierce, Cellular convection in polymer coating - an assessment, Ind. Eng. Chem. Prod. Res. Develop. 12 (1973) 67.

[8] J. Rudin, D.T. Wasan, Interfacial turbulence and spontaneous emulsification in alkali-acid oil systems, Chem. Eng. Sci. 48 (1993) 2225.

[9] C.A. Miller, Spontaneous emulsification produced by diffusion - a review, Colloid Surf. 29 (1988) 89.

[10] D. Myers, Surface, Interfaces and Colloids: Principles and Applications, VCH, New York, 1991.

[11] K.J. Ruschak, C.A. Miller, Spontaneous emulsification in ternary systems with mass transfer, Ind. Eng. Chem. Fundam. 11 (1972) 534.

[12] T. Niwa, H. Takeuchi, T. Hino, N. Kunou, Y. Kawashima, Preparation of biodegradable nanospheres of water-soluble and insoluble drugs with D, L-lactide/glycolide copolymer by a novel spontaneous emulsification solvent diffusion methanol, and the drug release behavior, J. Controlled Release 25 (1993) 89.

[13] A.J. Reuvers, Membrane formation: diffusion induced demixing processes in ternary systems, $\mathrm{Ph}$. D. Thesis, Twente University of Technology, The Netherlands, 1987.

[14] M.J. Han, D. Bhattacharyya, Changes in morphology and transport characteristics of polysulfone membranes prepared by different demixing conditions, J. Membr. Sci. 98 (1995) 191.

[15] H.C. Park, Y.P. Kim, H.Y. Kim, Y.S. Kang, Membrane formation by water vapor induced phase inversion, J. Membr. Sci. 156 (1999) 169.

[16] C.K. Ober, M.L. Hair, Effect of temperature and initiator levels on the dispersion polymerization of polystyrene, J. Polym. Sci. Part A 25 (1987) 195.

[17] E.P. Jacobs, W.D. Leukess, Formation of an externally unskinned polysulfone capillary membrane, J. Membr. Sci. 121 (1996) 149.

[18] K.D. Chesmel, J. Black, Cellular responses to chemical and morphologic aspects of biomaterials 1 . A novel in vitro model system, J. Biomed. Mater. Res. 29 (1995) 1089. 